\title{
Peptostreptococcus Mycotic Pseudo-Aneurysm
}

\author{
Geoffrey Horlait*, Pierre Bulpa \\ Intensive Care Unit, Université catholique de Louvain, CHU UCL Namur, Avenue Gaston Therasse, Yvoir, Belgium \\ Email: *Geoffrey.horlait@uclouvain.be
}

How to cite this paper: Horlait, G. and Bulpa, P. (2017) Peptostreptococcus Mycotic Pseudo-Aneurysm. Case Reports in Clinical Medicine, 6, 59-63. https://doi.org/10.4236/crcm.2017.63006

Received: February 27, 2017

Accepted: March 27, 2017

Published: March 27, 2017

Copyright $\odot 2017$ by authors and Scientific Research Publishing Inc. This work is licensed under the Creative Commons Attribution International License (CC BY 4.0).

http://creativecommons.org/licenses/by/4.0/ (c) (i) Open Access

\begin{abstract}
Mycotic pseudo-aneurysm is a rare and potentially fatal sequela of bacteraemia. The injury results in a localized, irreversible dilatation due to destruction of the blood vessel wall by infection, usually with an aerobic bacterium. Anaerobic bacteria are uncommon. We report an unusual case of a 72-year-old Caucasian man who presented with a rapidly progressive mycotic pseudoaneurysm of the right subclavian artery due to an anaerobic Gram-positive coccus Peptoniphilus asaccharolyticus. Despite optimal treatment, combining antibiotics and surgery, mycotic pseudo-aneurysm has a poor prognosis. When surgery is not possible, antibiotics alone with or without the placement of an endovascular stent have been proposed but in such cases, mortality is very significant. Despite the availability of non-invasive imaging techniques, a strong clinical suspicion is essential for early diagnosis of a mycotic aneurysm. Clinicians must be aware of these important aspects of management.
\end{abstract}

\section{Keywords}

Microbiology, Anaerobic Pathogens, Mycotic Aneurysm, Peptostreptococcus

\section{Introduction}

Mycotic pseudo-aneurysm is a rare occurrence. According to one series, between $0.9 \%$ and $2.6 \%$ [1] of aneurysms are caused by infection.

The injury results in a localized, irreversible dilatation due to destruction of the vessel wall. The infection may be localized or systemic. The difference between pseudo-aneurysm and true aneurysm is that the former partially compromises the vessel wall while the latter affects the entire structure. Pseudo-aneurysms occur most often in the abdominal aorta, femoral arteries, and mesenteric arteries and less frequently in the carotid, brachial and iliac arteries. They have also been described in the left ventricle and coronary arteries. The infection can be caused by septic embolisms to the vasa vasorum, a contiguous infective focus, direct bacterial inoculation by a penetrating trauma or bacteremic seeding of an existing in- 
jury (such as intimal injury, atherosclerotic plaque, or non-mycotic aneurysm).

The primary etiology is mainly bacterial in spite of the term "mycotic" originally used by Osler, who observed "fresh fungus-like vegetations". The microorganisms having the greatest affinity for arterial walls are Staphylococcus epidermidis, Salmonella species and Streptococcus pneumoniae [2]. Treponema pallidum and Mycobacterium tuberculosis are also frequently reported in emerging countries. This list is not exhaustive but other organisms are rarely encountered.

\section{Case Presentation}

A 72-year-old Caucasian man was admitted to the emergency room for management of right hemifacial and shoulder pain that had lasted for ten days with no other symptoms. His medical history included a triple coronary artery bypass graft performed ten years earlier, a pacemaker implanted for four years due to a high grade atrio-ventricular block and un-treated chronic obstructive pulmonary disease (classified as GOLD IV) due to smoking. More recently he had undergone a left iliofemoral bypass two weeks before and a diagnostic cystoscopy for hematuria during his hospital stay.

Clinical examination and laboratory tests were unremarkable but the patient was hospitalized due to a temperature of 38.2 degrees Celsius. Blood cultures were positive for a multi-drug susceptible Peptoniphilus asaccharolyticus (Peptostreptococcus species). Transthoracic echocardiography revealed thickening of the pace-maker leads and chest computerized tomography (CT) showed an abscess surrounding the arterial and venous brachiocephalic junction (Figure 1). The pacemaker leads were removed without complication ten days after symptoms

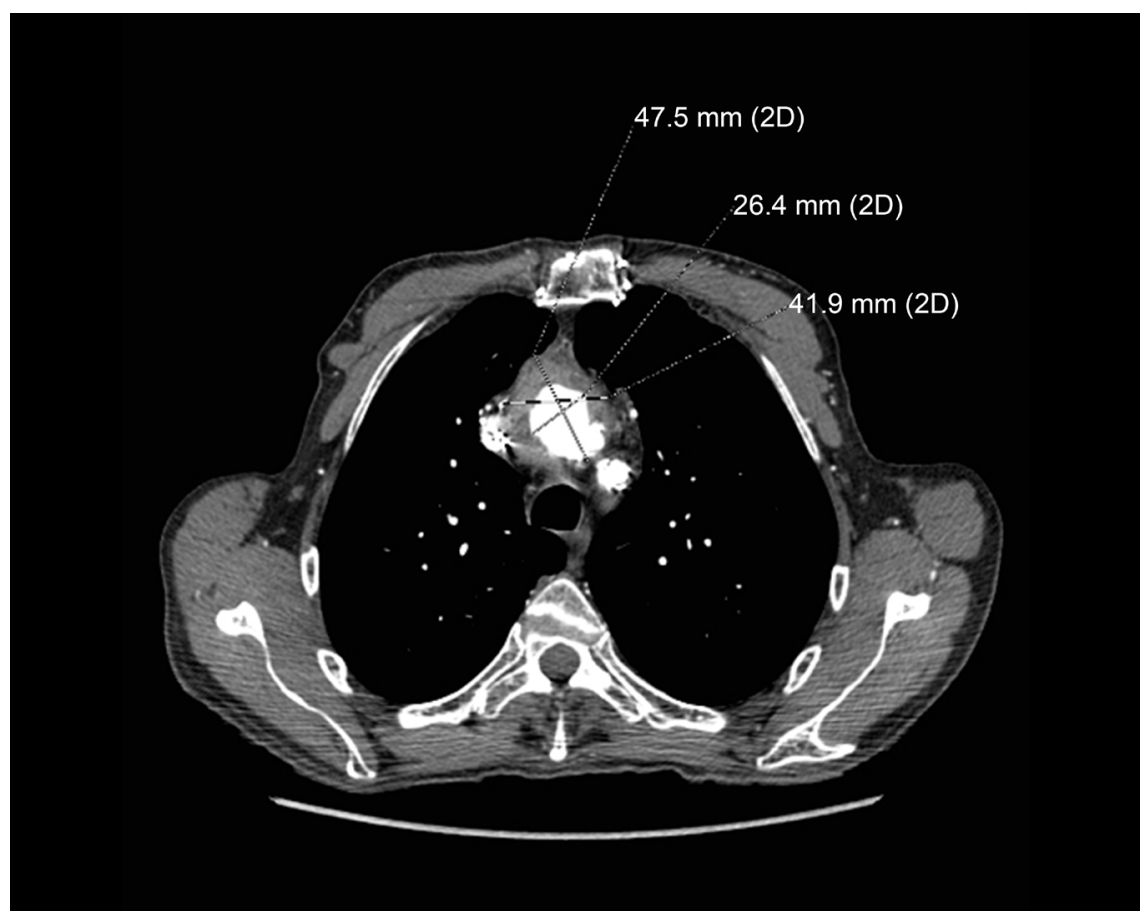

Figure 1. CT scan showing an abscess surrounding the arterial and venous brachiocephalic junction. 
appeared. No macroscopic sign of infection around the pacemaker box was observed and transesophageal echocardiography did not reveal any abnormality. Intravenous amoxicillin-clavulanic acid was initiated. The immediate postoperative period was uneventful.

On day 20 after symptoms appeared, the patient was admitted to the ICU after a rapidly reversible sudden alteration of consciousness after getting up from bed; no arrhythmia was noted on monitoring telemetry. Magnetic resonance imaging showed recent ischemic bilateral cerebral frontal and right temporal lesions. Routine chest radiography was performed.

On day 23, chest radiography (Figure 2) revealed a significant increase of the right upper mediastinum compared with its appearance on ICU admission. Clinical and radiological thoracic collateral venous circulation was observed suggesting Pemberton's sign. In addition, chest CT-scan showed a large mycotic pseudo-aneurysm at the origin of the arterial brachiocephalic trunk with a laminated superior vena cava and the two brachiocephalic veins (Figure 3).

Penicillin, gentamicin and flucloxacilline were started immediately and surgery was undertaken. Unfortunately, the patient died during the operation secondary to refractory heart failure complicated by a major pulmonary edema $\nearrow$. Intraoperative samples remained sterile. The family did not give permission for an autopsy.

The patient and his family provided informed consent to report his case. This study was conducted in accordance with the Declaration of Helsinki.

\section{Discussion}

Mycotic pseudo-aneurysm is rarely encountered and the most commonly cultured organisms are Staphylococcus epidermidis, Salmonella species and Treponema pallidum [2]. Peptoniphilus asaccharolyticus has, to our knowledge, never been reported as the cause of a case of mycotic pseudo-aneurysm. Peptonophilus asaccharolyticus, an anaerobic Gram-positive coccus, is part of the commensal flora found on all muco-cutaneous surfaces and is frequently isolated from deep organ abscesses, obstetric and gynaecological sepsis and intraoral infections. Some

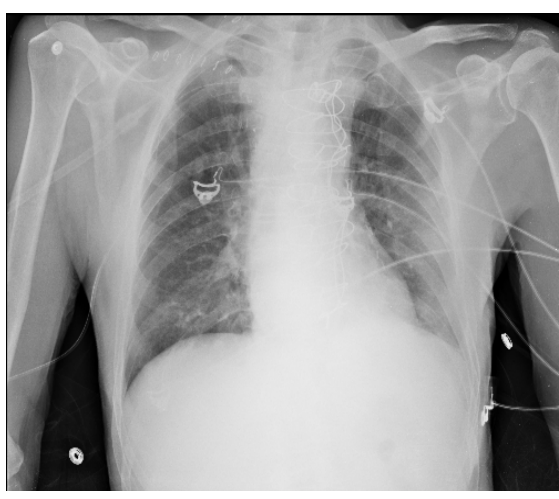

(a)

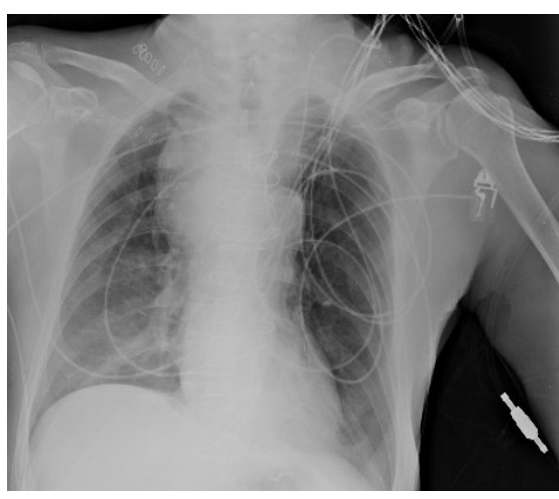

(b)

Figure 2. (a) Chest radiography on admission to the ICU; (b) Chest radiography at Day 23 showing a significant increase of the right upper mediastinum. 


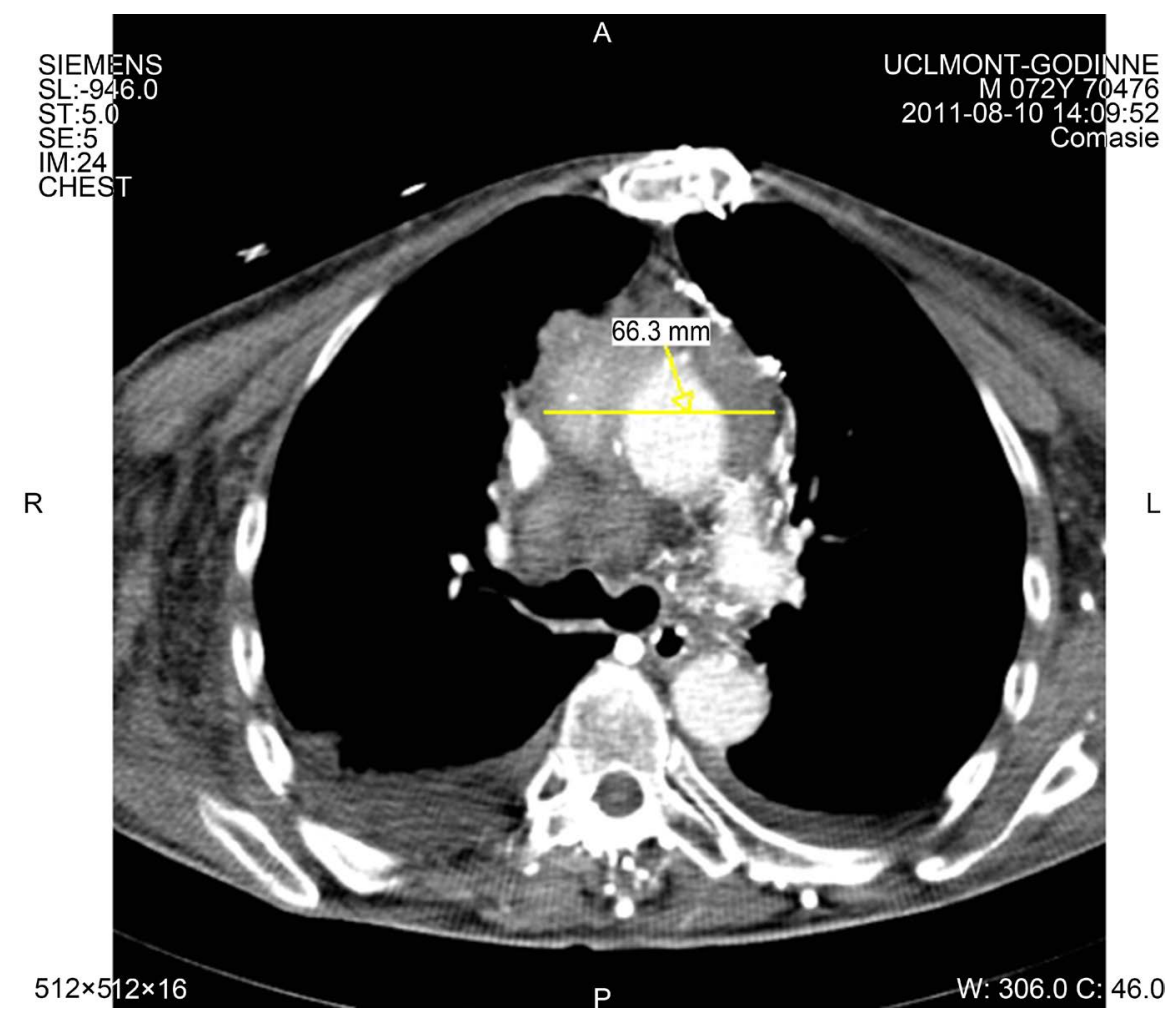

Figure 3. CT scan showing a large mycotic pseudo-aneurysm at the origin of the arterial brachiocephalic trunk with a laminated superior vena cava and the two brachiocephalic veins.

osteo-articular infections have also been associated with $P$. asaccharolyticus.

The clinical manifestations depend on the site of the pseudo-aneurysm. The classic presentation is a painful, pulsatile, and often enlarging mass with associated systemic features of infection such as fever (present in 88\% [3] of cases).

Pemberton's sign [4] reflects a compression of the jugular-venous subclavian axis and is characterized by facial congestion and positional cyanosis if the patient raises both arms above the head for 60 seconds. Swallowing disorders, dyspnea or stridor can appear or get worse during the test. This sign is typically secondary to diving thyroid goitre but may occur in any other extrinsic compressions. However, to our knowledge, no published reports have mentioned an association between Pemberton's sign and brachiocephalic trunk pseudo-aneurysm.

The gold standard for diagnosing mycotic pseudo-aneurysm is CT-scan with contrast injection [5]. Blood cultures allow isolation of the causative organism in $50 \%$ to $80 \%$ of cases [2].

Although no randomized studies have been performed, open surgical repair is the usual treatment combined with antibiotic therapy (for at least four weeks). When a mycotic pseudo-aneurysm involves the ascending aorta, the most appropriate therapy is replacement of the aorta with a homograft.

Without optimal treatment, mycotic pseudo-aneurysm is always fatal. When surgery is not possible, antibiotics alone with or without the placement of an endovascular stent have been proposed but in such cases, mortality is very significant 
[6]. In our case, despite a medical treatment, we were able to observe a rapid progression of the disease. The outcome of a surgery depends on many factors such as the anatomic site of the lesion and the patient's general condition. Classic surgical principles include wide debridement of infected tissue and extraanatomic bypass. Reported mortality from this approach exceeds $11 \%$ in the largest series [7]. Recent work has suggested that in situ grafting may be appropriate. Endovascular repair may also be feasible in a safe treatment option for patients who are in good general condition [3].

\section{Conclusion}

To conclude, despite the availability of non-invasive imaging techniques, a strong clinical suspicion is essential for early diagnosis of a mycotic aneurysm. Clinicians must be aware of these important aspects of management.

\section{Contributions}

Geoffrey Horlait and Pierre Bulpa contributed equally to this work.

\section{References}

[1] Gomes, M.N., Choyke, P.L. and Wallace, R.B. (1992) Infected Aortic Aneurysms. A Changing Entity. Annals of Surgery, 215, 435-442. https://doi.org/10.1016/j.revmed.2006.08.002

[2] Revest, M., Decaux, O., Cazalets, C., Verohye, J.P., Jégo, P. and Grosbois, B. (2007) Thoracic Infectious Aortitis: Microbiology, Pathophysiology and Treatment. La Revue de Médecine Interne, 28, 108-115. https://doi.org/10.1016/j.revmed.2006.08.002

[3] Soravia-Dunand, V.A., Loo, V.G. and Salit, I.E. (1999) Aortitis Due to Salmonella: Report of 10 Cases and Comprehensive Review of the Literature. Clin Infect Dis, 29, 862-868. https://doi.org/10.1086/520450

[4] Pemberton, H.S. (1946) Sign of Submerged Goitre. The Lancet, 248, 509. https://doi.org/10.1016/S0140-6736(46)91790-4

[5] Lee, W.K., Mossop, P.J., Little, A.F., Fitt, G.J., Vrazas, J.L., Hoang, J.K. and Hennessy, O.F. (2008) Infected (Mycotic) Aneurysms: Spectrum of Imaging Appearances and Management. Radio Graphics, 28, 1853-1868. https://doi.org/10.1148/rg.287085054

[6] Leon Jr., L.R. and Mills Sr., J.L. (2010) Diagnosis and Management of Aortic Mycotic Aneurysms. Vascular and Endovascular Surgery, 44, 5-13. https://doi.org/10.1177/1538574409344225

[7] Yu, S.Y., Hsieh, H.C., Ko, P.J., et al. (2011) Surgical Outcome for Mycotic Aortic and Iliac Anuerysm. World Journal of Surgery, 35, 1671-1678.

https://doi.org/10.1007/s00268-011-1104-9

\section{Abbreviations}

\section{CT: Computerized Tomography}


Submit or recommend next manuscript to SCIRP and we will provide best service for you:

Accepting pre-submission inquiries through Email, Facebook, LinkedIn, Twitter, etc. A wide selection of journals (inclusive of 9 subjects, more than 200 journals)

Providing 24-hour high-quality service

User-friendly online submission system

Fair and swift peer-review system

Efficient typesetting and proofreading procedure

Display of the result of downloads and visits, as well as the number of cited articles Maximum dissemination of your research work

Submit your manuscript at: http://papersubmission.scirp.org/

Or contact crcm@scirp.org 